Scientific Visualization, 2019, volume 11, number 4, pages 66 - 80, DOI: 10.26583/sv.11.4.06

\title{
Visualization Algorithms for Multi-criteria Alternatives Filtering
}

\author{
A.A. Zakharova ${ }^{1, A, B}$, D.A. Korostelyover,B, O.N. Fedonin'3,B \\ A Keldysh Institute of Applied Mathematics Russian Academy of Sciences \\ B Bryansk State Technical University \\ 1 ORCID: 0ooo-0oo3-4221-7710, zaa@tu-bryansk.ru \\ 2 ORCID: 0000-0002-0853-7940, nigm85@mail.ru \\ 3 ORCID: 0000-0002-3511-699X, rector@tu-bryansk.ru
}

\begin{abstract}
The article considers a decision-making problem for multi-criteria problems characterized by a large number of criteria and alternatives. As one of stages in making decisions in such situations, it is proposed to use a filtering of alternatives based on their visual images. For these purposes, the paper describes main aspects of this approach, as well as a statement of the algorithms for constructing the corresponding visual images based on radar and radial diagrams. A description of data preparation for operation of visualization algorithms for multi-criteria alternatives with an aim of their filtering is given. The paper describes software "AlternativesVisualizer" that implements the proposed algorithms, as well as providing interaction with an expert for visual filtering of multi-criteria alternatives. Additionally, the capabilities of the developed software are described. They include filtering alternatives based on threshold values, as well as the possibility of conducting a series of experiments in order to obtain the union or the intersection of filtered sets of alternatives. An experimental study of the developed algorithms and approaches effectiveness based on a synthetic test and an experiment on visual filtering of real alternatives describing the accuracy of calculating the inviscid flow around a cone using several OpenFoam solvers (rhoCentralFoam, pisoCentralFoam, sonicFoam, rhoPimpleFoam, QGDFoam) is presented. Each solver is characterized by 288 criteria, and with the help of the developed algorithms during visual filtering, the clear predominance of two solvers over the others is established.
\end{abstract}

Keywords: choice set visualization, alternative visual image, visual filtering, multicriteria alternatives, radial diagrams, radar charts. 


\section{Introduction}

During making decisions in various fields, managers of different levels often have to take into account a large number of various factors, as well as taking into account external conditions. Often these decisions are made intuitively and are based mainly on the experience and knowledge of the decision maker (DM). However, this is not the only way to make decisions. In modern science, a wide variety of decision making methods based on specialized approaches and algorithms are widely used [1]. At the same time, the solution of multicriteria decision making problems by means of these specialized approaches and algorithms can be not very effective when there are dozens and hundreds of alternatives, and they all have more than a dozen criteria. These situations are quite common when, for example, the source of alternatives is multisensory systems [2], or when initially many alternatives are formed by means of specialized systems in the course of multiple simulation $[3,4]$.

Therefore, in such cases, the initial choice set is firstly filtered, and then decision making methods are used already on a filtered selection of alternatives. Traditionally, statistical methods are used for filtering. Taking into account the fact that during decision making DM activates his mental activity, the same factor can be used to solve the problem of filtering alternatives. To do this, DM's mental activity can be addressed to a comparative analysis of alternative visual images (visual representations of vector criteria) [5]. But it is necessary to develop effective algorithms for visual filtering of alternatives, so that the main DM's efforts should be focused exactly on intelligent visual selection, and not on accompanying actions or calculations.

\section{Algorithm of alternatives' visualization in multi- criteria decision making problems}

In multi-criteria decision making problems, the criteria characterizing alternatives can be set both as quantitative and qualitative characteristics. In order to work with various criteria, first it is necessary to reduce them to numeric variables.

A wide range of different algorithms and methods are used for these purposes [1]. These methods and algorithms allow to convert the initial values of the criteria into numerical values in the form of the corresponding functions $f_{i}(k)$, where $1 \leq i \leq K, K$ is the number of criteria. Function $f_{i}(k)$ is usually nonlinear and may contain additional conditions for different intervals of $k$ initial values of $i$-criterion. The dimension (i.e. range of possible values) of $f_{i}(k)$ function for different criteria may vary significantly. For this reason, for further work with alternative visual images having these criteria, it is necessary to normalize $f_{i}(k)$ functions. One of the traditional approaches in this case is normalization by interval [0; 1$]$ based on the maximum and minimum possible values of the function.

1. $f_{i}^{\prime}(k)=\frac{f_{i}(k)-f_{i, \min }}{f_{i, \max }-f_{i, \min }}$, if the maximum criterion value corresponds to the best option;

2. $f_{i}^{\prime}(k)=\frac{f_{i, \max }-f_{i}(k)}{f_{i, \max }-f_{i, \min }}$, if the minimum criterion value corresponds to the best option.

Values $f_{i, \min }$ and $f_{i, \max }$ are defined in the given choice set $A=\left\{A_{j}\right\}, 1 \leq j \leq N, A_{j}=\left\{f_{i}\left(k_{i, j}\right)\right\}$, where $N$ is a number of alternatives either is from valid (anticipated) values, or is defined according to the formulas:

$$
\begin{aligned}
& f_{i, \min }=\min \left(f_{i}\left(k_{i, j}\right)\right), \\
& f_{i, \max }=\max \left(f_{i}\left(k_{i, j}\right)\right) .
\end{aligned}
$$


Various methods are currently used to visualize many alternatives [6]: parallel coordinates plot (Fig. 1), bar diagrams (Fig. 2), radar diagrams ([7], Fig. 3), pie charts (Fig. 4) and others.

Most of these approaches focus on displaying several alternatives in a single chart (Fig. 1-3). This way is quite appropriate when the number of alternatives (about 3-10) and criteria (about 3-7) is not big. However, such visualization methods are not suitable when you have to analyze dozens and hundreds of alternatives, each of which can have more than 10 criteria, because the chart becomes too overloaded and complex to analyze. A large number of alternatives can be visualized, for example, by using a set of pie charts, each representing a different alternative (Fig. 4). However, effective filtering of alternatives requires a more holistic perception of their visual image. Pie charts do not sufficiently provide such a perception with a large number of criteria due to color diversity.

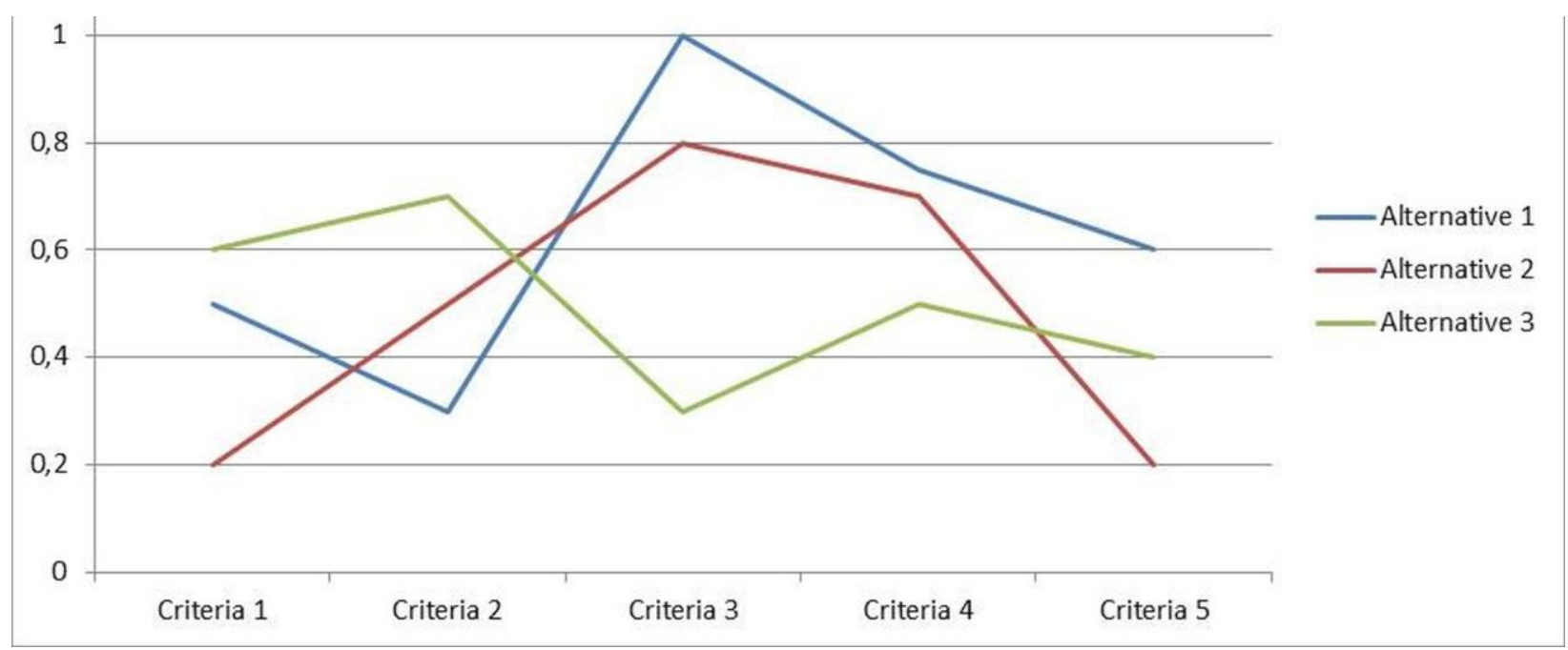

Fig. 1. Alternatives' visualization in the form of polyline criteria values

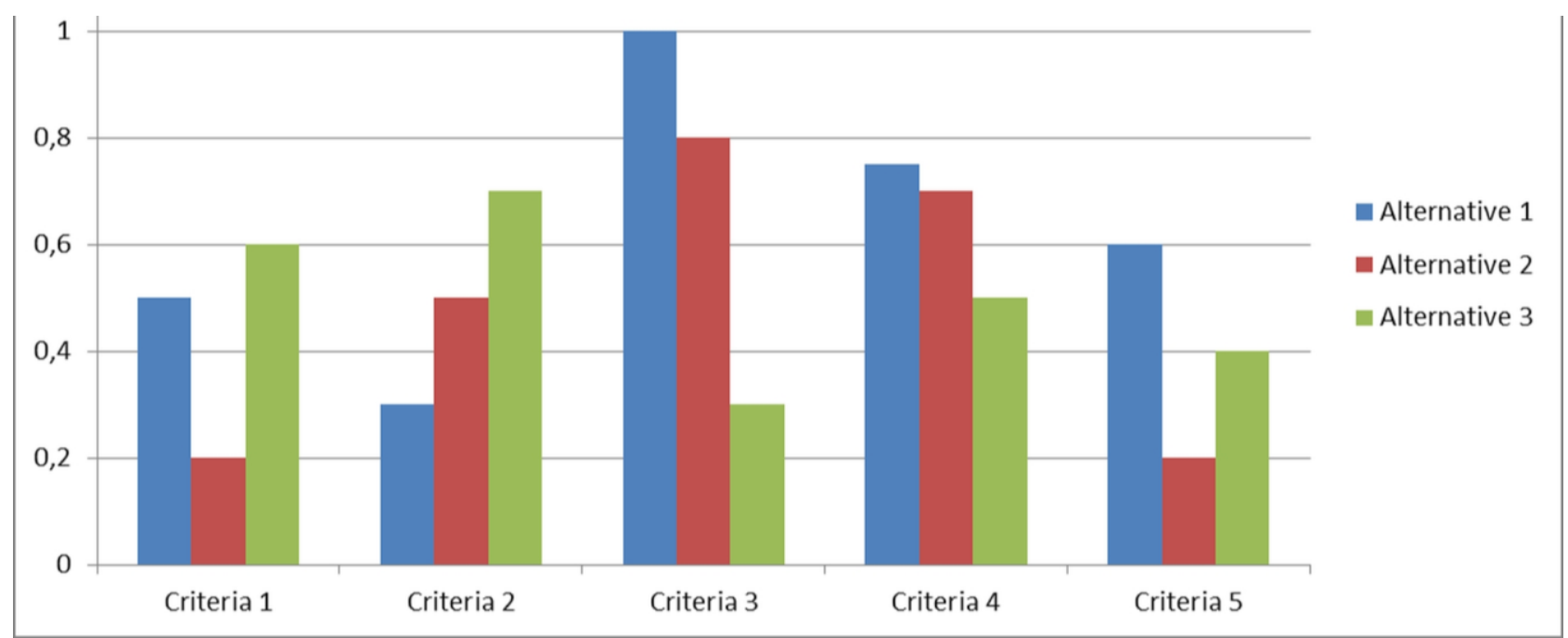

Fig. 2. Alternatives visualization in the form of bar diagrams 


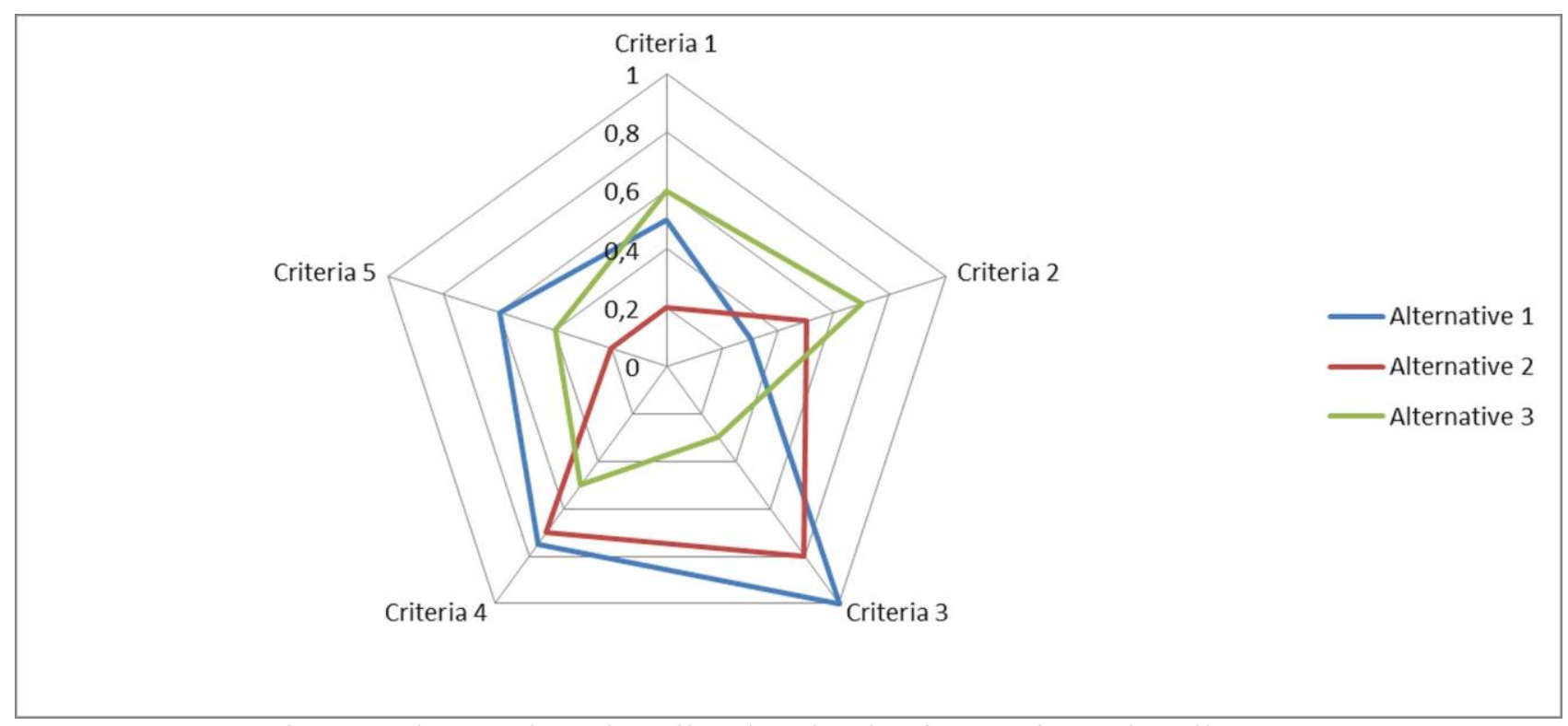

Fig. 3. Alternative visualization in the form of a radar diagram
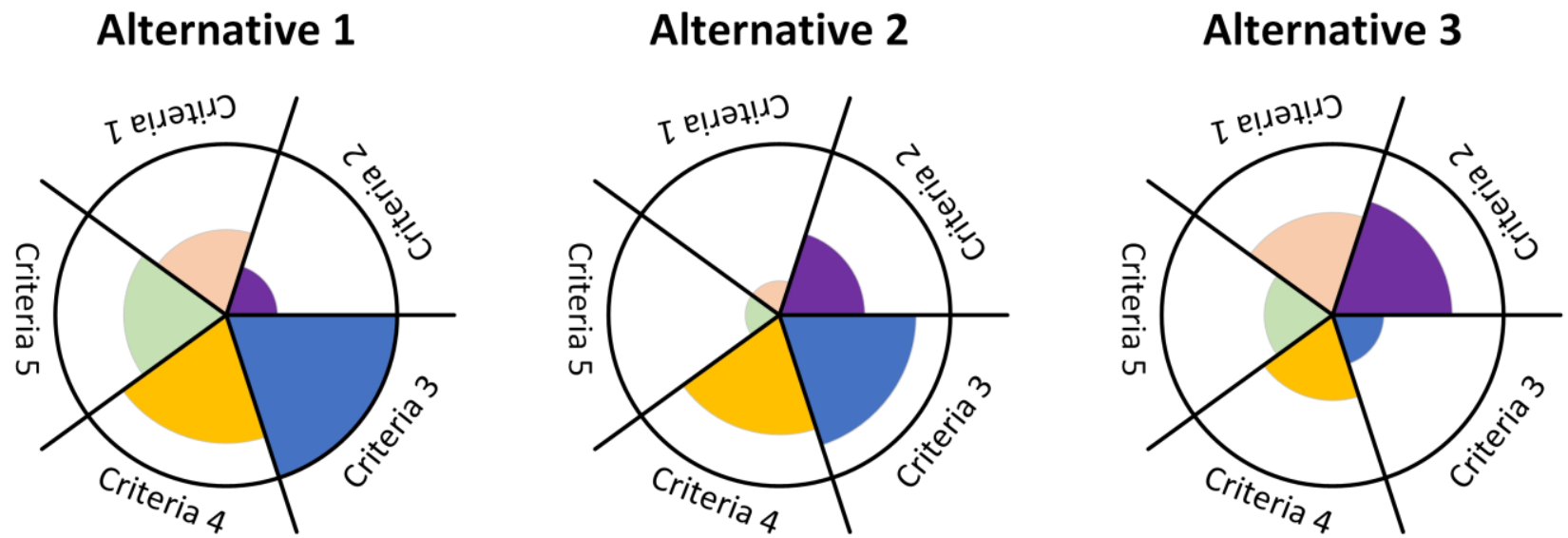

Fig. 4. Alternatives visualization in the form of pie charts

Taking into account the considered peculiarities we formulate the main criteria of constructing an algorithm for visualizing alternatives in multi-criteria decision making problems in order to filter them.

1. Each alternative should be represented as a single image.

2. Since there may be too many alternatives, it is necessary to provide a mechanism for focusing on a small sample of them and the possibility of changing this focus.

3. To highlight equivalent criteria with a different color is inappropriate, because the color can adversely affect DM's alternative.

4. Color effect is useful when visualizing alternatives for criteria values close to optimal in order to further focus DM's attention on them.

Based on these criteria we proposed an appropriate algorithm. Within this algorithm, two main aspects can be distinguished.

1. Visualization of one alternative.

2. Allocation of alternatives and focusing method on their subset (focus subset).

The construction of a visual image is based on pie charts and radar diagrams (Fig. 3, 4). Their common feature is that the value of the alternative by a separate criterion is located on a separate beam (radar diagram) or a sector of the circle (pie chart). However, moving away from a traditional representation of radar diagrams, we will place each alternative on a separate circle (as in pie charts). 
For an alternative to be represented as a single image, it is advisable to use a single filling style for all criteria: for the pie chart, the corresponding sectors are filled, and for the radar diagram, the corresponding polygon is filled. Taking into account that the sector radius in the pie chart and the position of polygon points is determined by the proximity of the normalized criterion value of the corresponding alternative to 1 (the closer to one, the better), it is advisable to use a gradient radial fill: in the center of the circle the color is neutral, and close to the border it is contrast (for example, red).

In addition to the color effect in this approach, an additional source of focusing and choice preferences among alternatives is the area of the corresponding figure.

1. For a pie chart, the shape area is defined as a sum of sector areas:

$$
S_{j}=\sum_{i=1}^{K} S_{j, i}
$$

where $S_{j, i}=\frac{\pi \cdot\left(f_{i}^{\prime}\left(k_{i, j}\right)\right)^{2}}{K}$ in the case, if all the sectors have the same angle (this is usually the case where all criteria are equal) and radius of sector is equal to normalized value of corresponding criterion (algorithm "Sectors (radius)"). If, however, there is some preference concerning criteria, then a sector with a larger angle can be specified for the more preferred criteria. Let us denote this angle as $\alpha_{i}$ (radian), then

$$
S_{j, i}=\frac{\left(f_{i}^{\prime}\left(k_{i, j}\right)\right)^{2} \cdot \alpha_{i}}{2}
$$

The value of $\alpha_{i}$ angle can be defined on the basis of ranking algorithms or weighting criteria used in decision making methods [1]. This approach has a peculiarity that the sector area is proportional to the square of the alternative value by the criterion, which may unnecessarily draw attention to the alternatives that have the maximum value of one of the criteria. To reduce this degree of influence, it is possible to establish not a quadratic, but a linear dependence between the sector area and the alternative value according to the corresponding criterion: $S_{j, i}=\frac{f_{i}^{\prime}\left(k_{i, j}\right) \cdot \alpha_{i}}{2}$. Then the radius of the corresponding sector will be defined by the formula: $r_{j, i}=\sqrt{f_{i}^{\prime}\left(k_{i, j}\right)}$ (algorithm "Sectors (radius root)").

2. For a radar diagram, the area of a shape is defined as the sum of the areas of triangles:

$$
S_{i}=\sum_{i=1}^{K} S_{j, i}
$$

where $S_{j, i}=\frac{f_{i}^{\prime}\left(k_{i, j}\right) \cdot f_{i+1}^{\prime}\left(k_{i+1, j}\right) \cdot \sin \left(\alpha_{i}\right)}{2}, f_{K+1}^{\prime}\left(k_{K+1, j}\right)=f_{1}^{\prime}\left(k_{1, j}\right)$.

The main peculiarity of calculating the area of the polygon according to this formula is that its final value is in addition affected by the order of criteria placement, because when placing nearby criteria with large values $f_{i}^{\prime}\left(k_{i, j}\right)$, then the total area is larger, which means that such alternatives are more focused on themselves attention of DM than others. On the other hand, the focusing factor in this case may also be a large number of sharp angles in the polygon. Therefore, for this type of diagrams let us consider two modifications of exchanging criteria:

- grouping a number of criteria with higher values for the displayed alternatives;

- alternation of criteria with higher and lower values for the displayed alternatives.

In both versions, we first calculate the mean value of $m_{i}$ for each criterion in the set of displayed alternatives $A^{\prime}=\left\{A_{t}\right\}$, where $1 \leq t \leq T, T$ is the number of displayed alternatives:

$$
m_{i}=\frac{\sum_{t=1}^{T} f_{i}^{\prime}\left(k_{i, t}\right)}{T} \text {. }
$$

Next, we sort descending $m_{i}$ with remembering the initial $i$ position. Let us present the result as a sequence

where $m_{i_{1}}=\max \left(m_{i}\right), m_{i_{K}}=\min \left(m_{i}\right)$.

$$
p_{0}=\left\{i_{1}, \ldots, i_{K}\right\},
$$


For the first modification (grouping), $p_{1}$ exchange is defined as follows (radar ( $p_{1}$ exchange)):

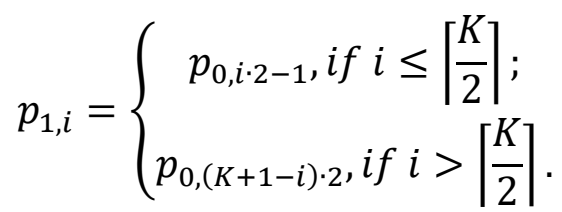

For the second modification (grouping), $p_{2}$ exchange is defined as follows (radar ( $p_{1}$ exchange)):

$$
p_{2, i}=\left\{\begin{array}{l}
p_{0,(i+1) / 2}, \text { if } i \text { is odd; } \\
p_{0, \frac{i}{2}+\left[\frac{k}{2}\right\rceil}, \text { if } i \text { is even }
\end{array}\right.
$$

For example, suppose we have two alternatives $A_{1}=\{1.0 ; 0.4 ; 0.8 ; 1.0\}$ and $A_{2}=\{0.5 ; 1.0$; $1.0 ; 0.2\}$. Average values of the criteria will be $\{0.75 ; 0.7 ; 0.9 ; 0.6\}$. Then $p_{o}=\{3 ; 1 ; 2 ; 4\}$ (i.e. maximum value we have for the 3 rd criterion, and minimal value for the 4 th). The permutation $p_{1}$ will be: $p_{1}=\{3 ; 2 ; 4 ; 1\}$, and $p_{2}=\{3 ; 2 ; 1 ; 4\}$. Those for $p_{1}: A_{1}\left(p_{1}\right)=\{0.8 ; 0.4 ; 1.0$; $1.0\}, A_{2}\left(p_{1}\right)=\{1.0 ; 1.0 ; 0.2 ; 0.5\}$, and for $p_{2}: A_{1}\left(p_{2}\right)=\{0.8 ; 0.4 ; 1.0 ; 1.0\}, A_{2}\left(p_{2}\right)=\{1.0 ; 1.0 ; 0.5$; $0.2\}$.

Building a visual image of alternatives in the case of using radar diagrams starts with the use of one of the two considered exchanges of criteria: $p_{1}$ or $p_{2}$.

When visual images of alternatives is placed on the screen, we follow these principles:

1. It is necessary to visualize all alternatives in a simplified form on a smaller part of the screen within a rectangle (simplified display). In this part of the screen you need to place the selection area of alternatives (it is also advisable to do this in the form of a rectangle). This area must be movable.

2. When you change the position of the selection area on the simplified display, you need to define a list of alternatives completely being inside it. These alternatives are displayed in the focus area, which is also a rectangular portion of the screen that takes up significantly more space than the simplified display area.

The position of alternatives in these areas is determined by the grid consisting of rows and columns (Fig. 5). The alternatives themselves are placed at the nodes of this grid. In this case, for a more uniform distribution of alternatives, the grid is not orthogonal, but with an offset in even rows by the circle radius in which the alternative is visualized.

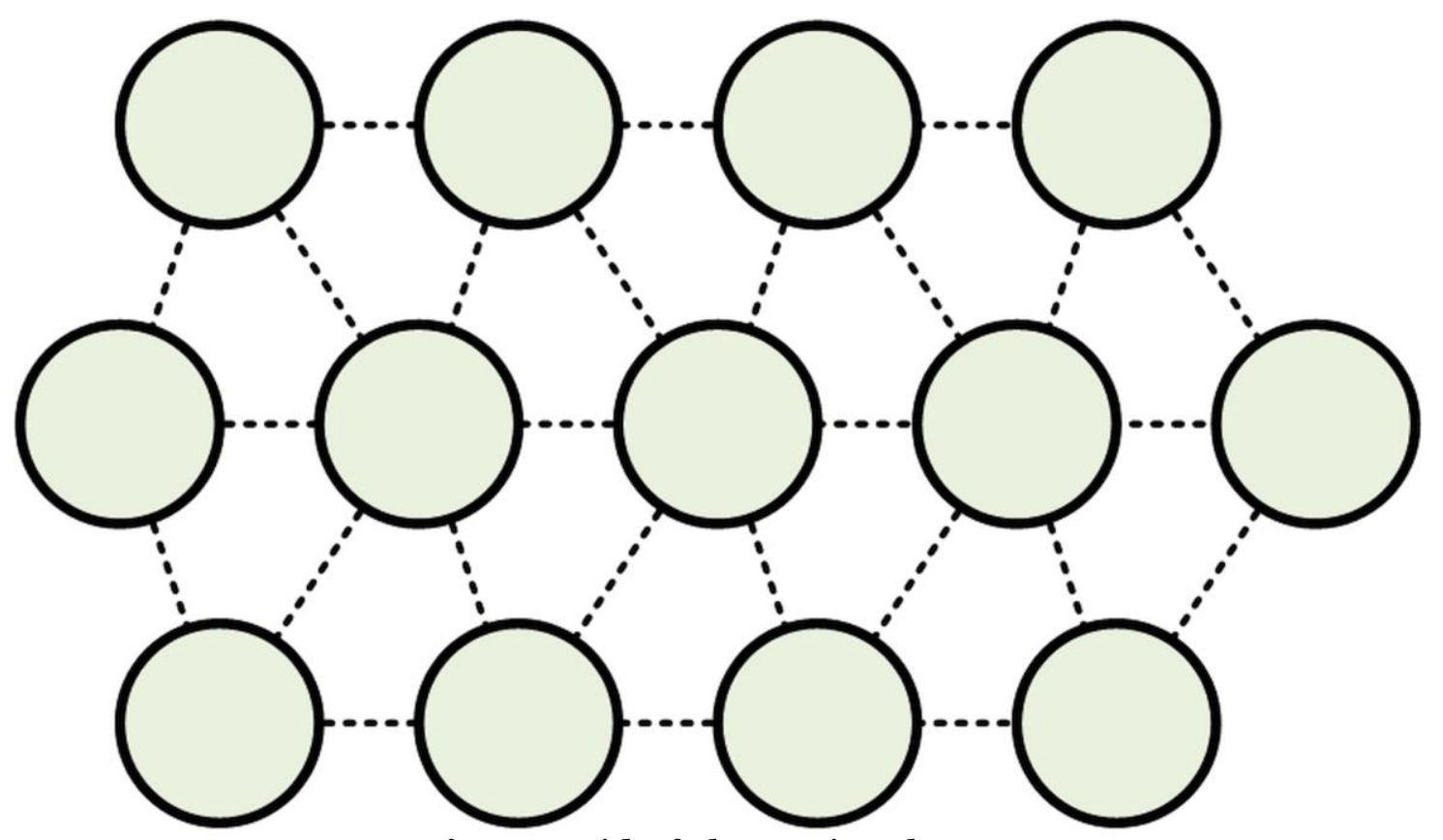

Fig. 5. Grid of alternatives layout 


\section{Description of the software for visual filtering of al- ternatives}

The algorithms described above were implemented in a special program "AlternativesVisualizer". The developed software allows to load from the table view a list of alternatives with a numerical representation of values according to the relevant criteria, as well as to filter them in the rendering mode (Fig. 6).

For filtering the program provides two approaches - manual filtering by hiding or displaying alternatives (the central part of the form in Fig. 6), as well as filtering based on the threshold values of the criteria (the right part of the form in Fig. 6) - all alternatives that do not meet the thresholds are hidden.

At the bottom of the form there is a simplified area of alternatives visualization where the user can select a range of alternatives to be displayed in the main area by moving the corresponding rectangular block. Using the toolbar buttons, the user can enter the hide or show alternatives mode. Hiding or displaying is done by clicking the mouse button on the corresponding alternative on the main visualization area.

The program "AlternativesVisualizer" supports all four visualization options considered in the paper:

- sectors with radii proportional to the criteria for the alternative;

- sectors with radii proportional to the roots of the criteria values for the alternative;

- radar diagram with $p_{1}$ criteria exchange;

- radar diagram with $p_{2}$ criteria exchange.

Working with the program, the user can perform several experiments on filtering alternatives by means of different ways of their visualization. The results are summarized in a table (Fig. 7).

In addition, on the basis of the table obtained, the program "AlternativesVisualizer" analyzes the results and displays the numbers of alternatives that the user has chosen in all experiments in a separate list. This approach allows one to reduce the resulting set of filtered alternatives further, leaving only those that the DM has preferred for all visualization methods.

With the help of the developed program "AlternativesVisualizer", an experiment was conducted. A choice set (200 alternatives) with 15 criteria was randomly generated. An alternative with maximum values for all criteria out of all 200 generated was added to this set (in order to verify that this alternative will not be filtered). Thus, there were 201 alternatives in total. The problem of filtering alternatives was solved 5 times using different methods: 4 methods of different visualization options and one method - setting low threshold values for all criteria in order to reduce the number of displayed alternatives by an order of magnitude. The results of the experiments are presented in table 1. 
$\approx$ Visual filtering of alternatives

File Alternatives Options Help

Table Visualization Results

$\infty$

$x$

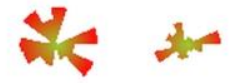

is

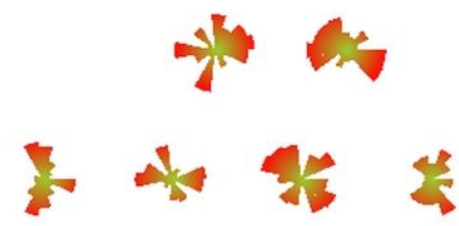

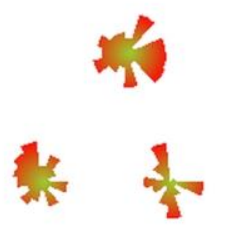

Criteria №2 279

$\checkmark$

Criteria №3

.

Citteria N24

.

Criteria №5

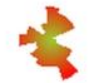

$\square$

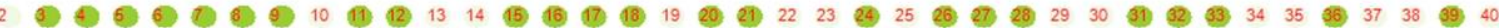

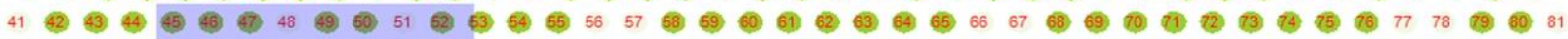

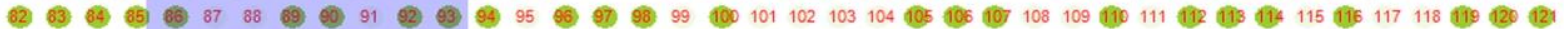

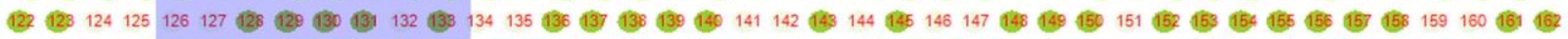

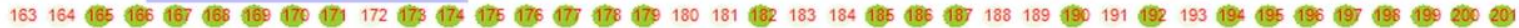

Fig. 6. Interface of software for visual filtering of alternatives "AlternativesVisualizer"

Table 1. Experiment results of filtering alternatives by using various methods.

\begin{tabular}{|c|c|c|c|}
\hline № & $\begin{array}{l}\text { Filtering } \\
\text { type }\end{array}$ & $\begin{array}{l}\text { Number of } \\
\text { alterna- } \\
\text { tives }\end{array}$ & Alternatives list \\
\hline & $\begin{array}{l}\text { Sectors (ra- } \\
\text { dius) }\end{array}$ & 41 & $\begin{array}{l}4,5,7,8,12,14,18,27,29,34,41,42,46,52,53,55,56, \\
62,76,79,82,83,89,97,103,106,110,120,127,130,146, \\
147,155,156,158,159,161,170,184,197,201\end{array}$ \\
\hline 2 & $\begin{array}{l}\text { Sectors (ra- } \\
\text { dius root) }\end{array}$ & 50 & $\begin{array}{l}4,5,7,14,17,18,19,21,27,29,33,34,36,41,42,46,53, \\
56,62,63,76,79,81,82,83,84,87,90,97,103,120,122, \\
127,130,132,133,138,139,141,146,147,154,155,156, \\
165,170,173,177,184,201\end{array}$ \\
\hline 3 & $\begin{array}{l}\text { Radar }\left(p_{1}\right. \\
\text { exchange })\end{array}$ & 45 & $\begin{array}{l}4,5,7,14,17,18,21,27,31,33,34,36,42,52,56,62,63, \\
76,79,83,84,89,90,97,103,106,118,120,121,130,141, \\
142,146,147,154,155,156,158,161,173,177,178,182, \\
184,201\end{array}$ \\
\hline 4 & $\begin{array}{l}\text { Radar (p } \\
\text { exchange) }\end{array}$ & 36 & $\begin{array}{l}4,5,7,14,17,18,19,21,27,29,33,34,36,52,53,55,62, \\
76,79,83,84,90,103,104,106,120,127,130,138,139, \\
146,147,154,155,177,201\end{array}$ \\
\hline & Threshold & 27 & $\begin{array}{l}4,5,27,36,39,42,43,50,5 \\
100,104,106,110,149,154,1\end{array}$ \\
\hline
\end{tabular}

Using the program (on the "Results" tab) we find the intersection of choice sets obtained in 1-4 experiments, and we get 18 alternatives (Fig. 7). When determining the intersection of choice sets obtained in all experiments, we obtain only 7 alternatives: $4,5,27,62,83,155$, 201. In the case of combining choice sets (this feature is also available in the "Results" tab of the program) obtained in experiments 1-4, we will get 68 alternatives. And if we combine choice sets obtained in all five experiments, we get 78 alternatives. 


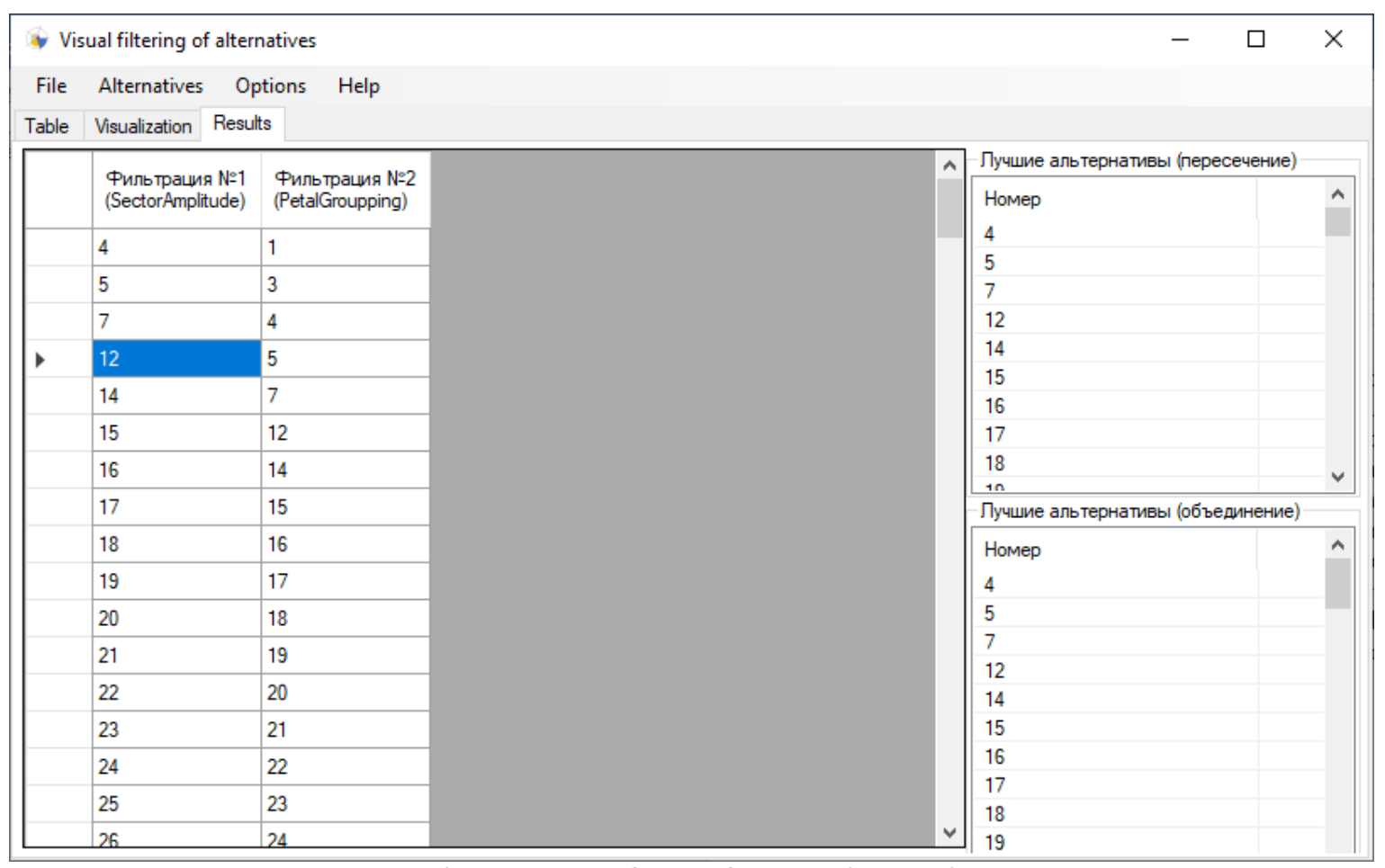

Fig. 7. Interface of «Results» tab

Analyzing the results obtained, we can conclude that the greatest effect of filtering alternatives is achieved by finding the intersection of sets obtained in experiments with different methods of visualization in combination with threshold filtering alternatives. Further work on selecting the optimal alternative should be carried out with this subset using other methods of decision making, designed and applicable on a small number of alternatives.

\section{Experiment}

With the help of the developed software "AlternativesVisualizer", there was also an experiment conducted for real alternatives. We got estimations according to 144 criteria for norms L1 and L2 for the computational problem of evaluating the accuracy of the calculations of inviscid flow around a cone by means of several OpenFoam solvers (rhoCentralFoam, pisoCentralFoam, sonicFoam, rhoPimpleFoam, QGDFoam) [8, 9].

After preliminary processing of the initial data according to norm L1, only 88 criteria were left (as for the rest of the criteria, the data were incomplete). Having constructed and analyzed visual images for different algorithms, we determined that the alternative corresponding to pisoCentralFoam solver is almost always occupies a large area and is more contrast by using the red fill on the border of the corresponding visual image, so we can conclude that this algorithm is the most preferable (Fig. 8-11). 

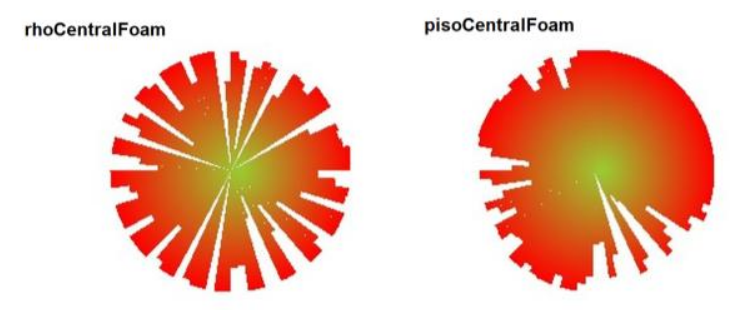

sonicFoam

rhoPimpleFoam
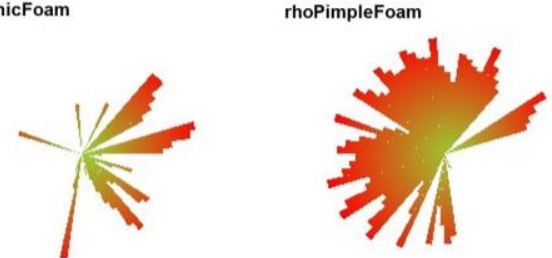

QGDFoam

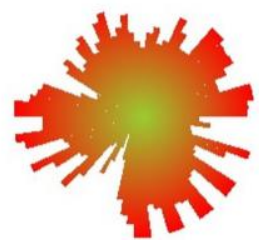

Fig. 8. Alternatives visualization using method "Sectors (radius)" for criteria on L1 norm
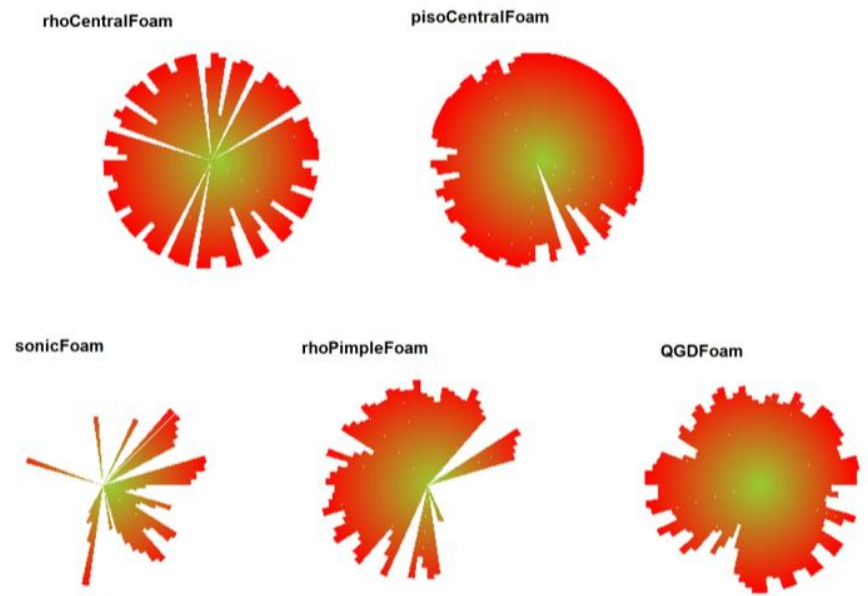

Fig. 9. Alternatives visualization using method "Sectors (radius root)" for criteria on L1 norm

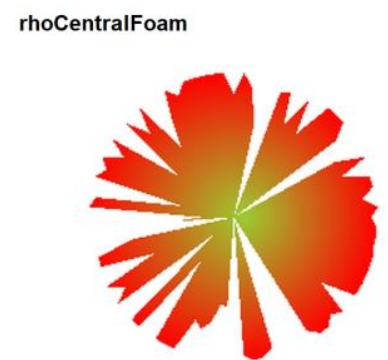

pisoCentralFoam

sonicFoam

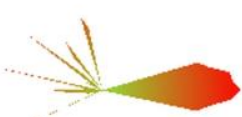

rhoPimpleFoam

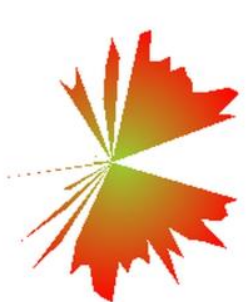

QGDFoam

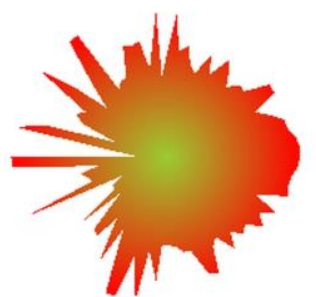

Fig. 10. Alternatives visualization using method "Radar ( $\mathrm{p}_{1}$ exchange)" for criteria on L1 norm 


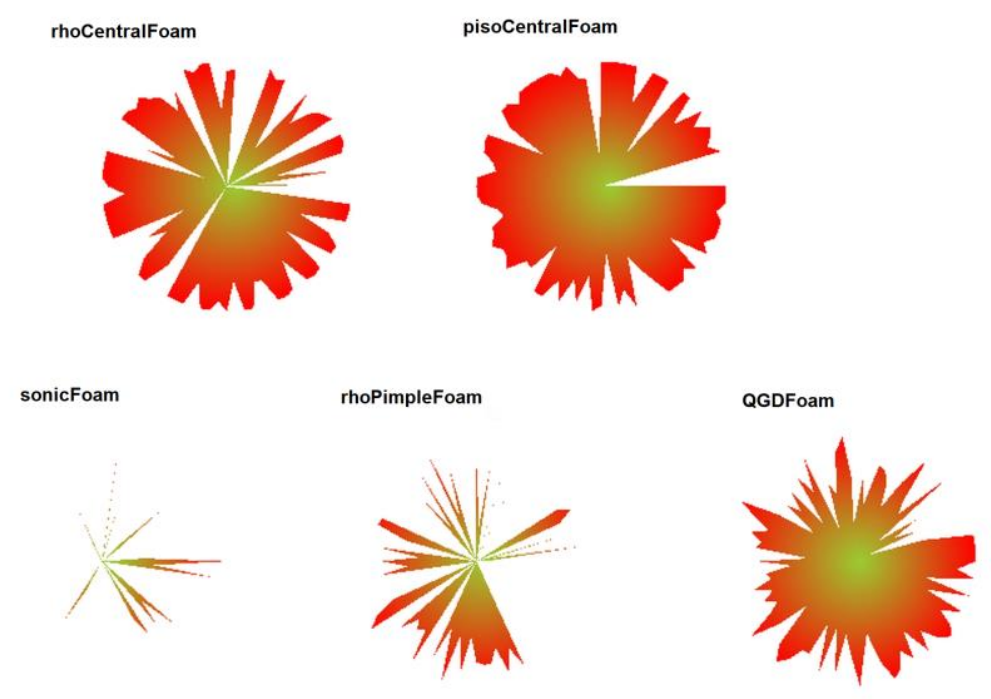

Fig. 11. Alternatives visualization using method "Radar ( $p_{1}$ exchange)" for criteria on L1 norm

176 criteria were selected when comparing the alternatives according to two norms, and one of the alternatives was excluded from consideration, as there were no its data on L2 norm. As a result of visual filtering, it was determined that from four alternatives the two alternatives have a large area, as well as more contrast (due to the use of red color at the borders of the visual image) - this visual images are determinate rhoCentralFoam and pisoCentralFoam solvers (Fig. 12-15). However, preference can again be given to pisoCentralFoam solver, because in almost all images it visually occupies a slightly larger area compared of rhoCentralFoam solver.
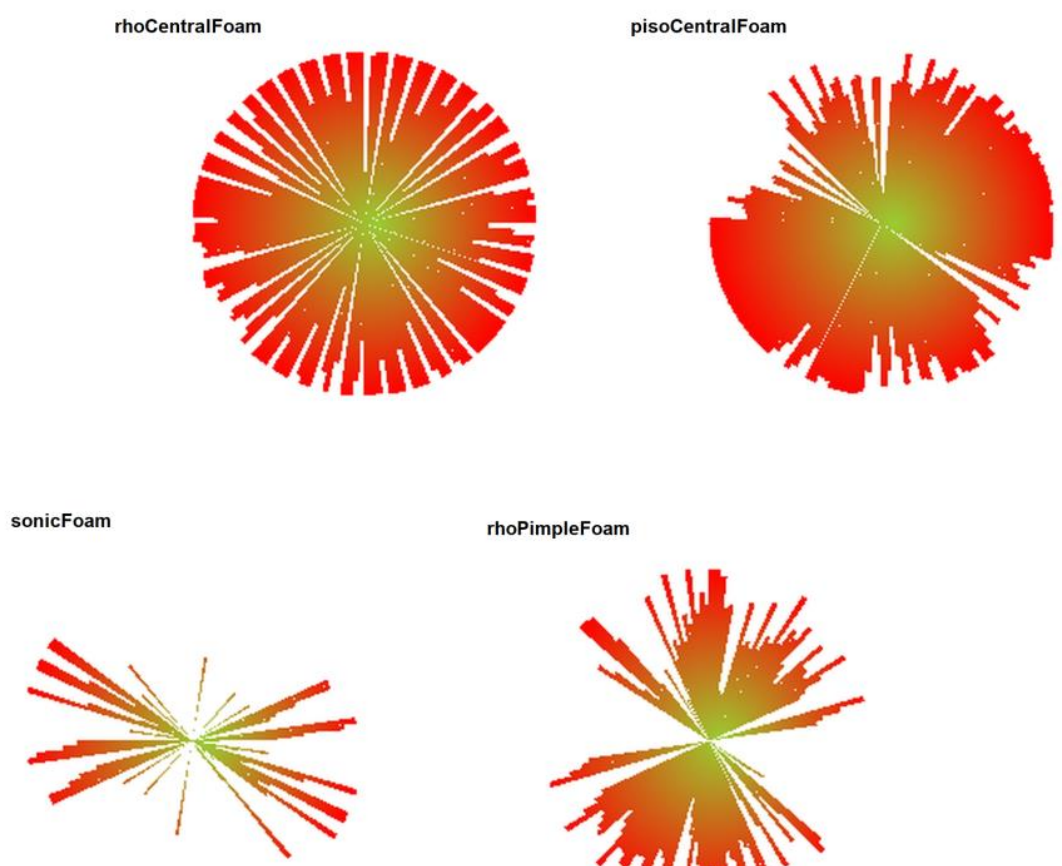

rhoPimpleFoam

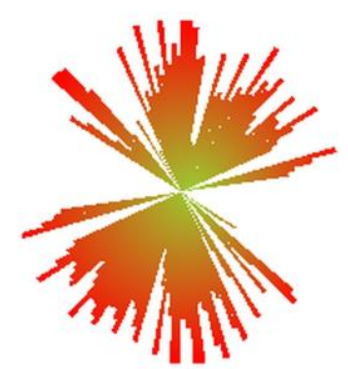

Fig. 12. Alternatives visualization using method "Sectors (radius)" for criteria on L1 and L2 norms 

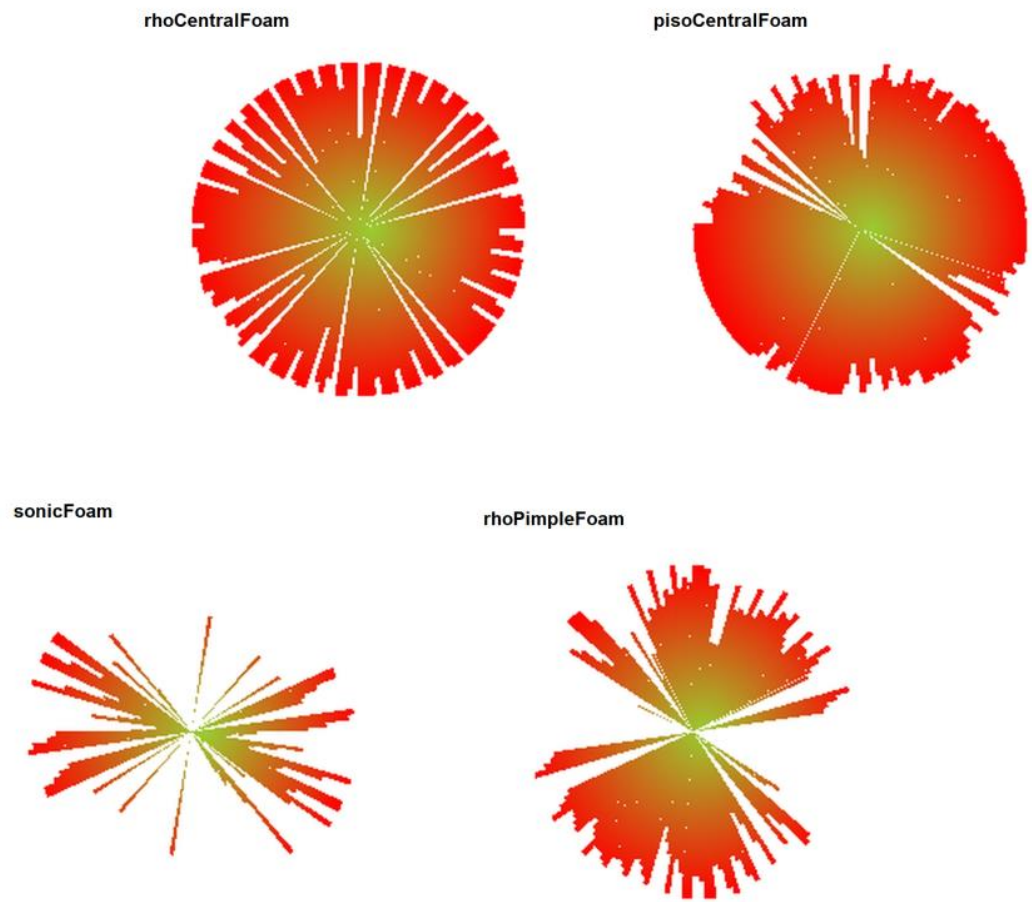

Fig. 13. Alternatives visualization using method "Sectors (radius root)" for criteria on L1 and L2 norms
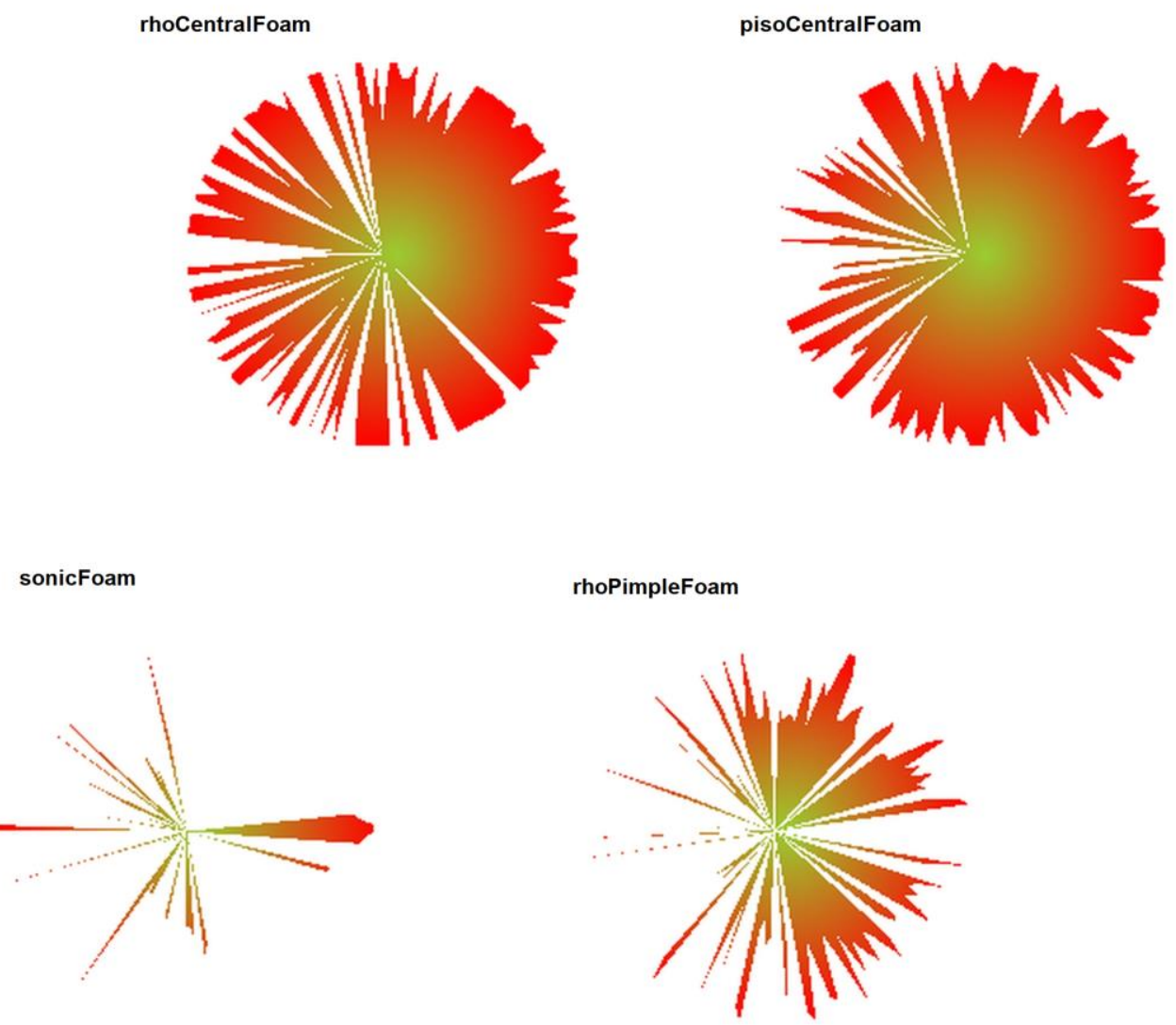

Fig. 14. Alternatives visualization using method "Radar ( $\mathrm{p}_{1}$ exchange)" for criteria on L1 and L2 norms 

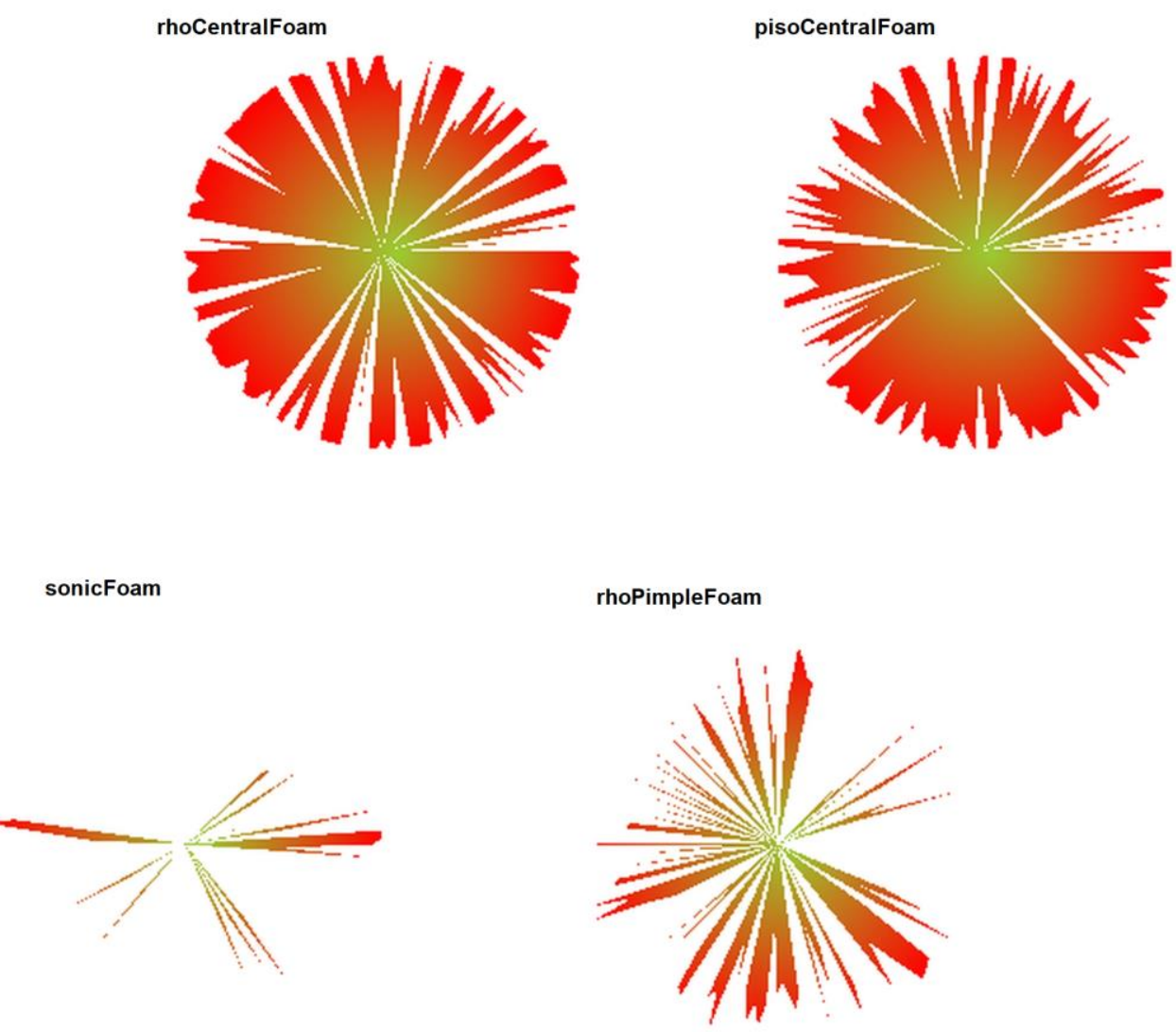

Fig. 15. Alternatives visualization using method "Radar ( $\mathrm{p}_{2}$ exchange)" for criteria on L1 and L2 norms

\section{Conclusion}

The approaches to placement and focusing alternatives and algorithms of constructing visual images for multi-criteria alternatives (four algorithms) considered in the paper have shown that visual filtering can be quite an effective method in decreasing the initial choice set. As a result of several experiments on filtration by means of the developed software an initial set of 201 alternatives has been reduced to 27-50 alternatives. Identifying a subset from the results of different experiments, which is common for the results of all experiments, allowed us to decrease this set to only 7 alternatives, i.e., to reduce the number by about 28 times.

Four ways of constructing visual images are considered in the paper. However, it is possible to expand these options further through the use of additional visualization techniques: $3 \mathrm{D}$ visualization, other types of diagrams, other types of defining criteria exchange, etc.

Also, an experiment was conducted on the visual selection of the best alternative (solver) according to the known criteria characterizing the accuracy of calculations. Comparison was made for 5 alternatives and the number of criteria was 176. According to the comparative visual analysis it was clearly seen that pisoCentralFoam solver gives more accurate calculation results.

An additional feature of the developed mathematical support and software is that it is suitable for using by a group of experts, each of whom can conduct a series of experiments with different types of constructing visual images, and then the results can be summarized.

\section{Acknowledgments}

The work was supported by Russian Science Foundation grant № 18-11-00215. 


\section{References}

[1] Figuera J., Greco S. and Ehrgott M. (Eds). Multiple Criteria Decision Analysis: State of the Art Surveys. - New York: Springer, 2005. - DOI: 10.1007/b100605.

[2] Zakharova A., Vekhter E., Shklyar A., Pak A. Visual modeling in an analysis of multidimensional data // Journal of Physics: Conference Series. - 944. - 012127, 2018. DOI: 10.1088/1742-6596/944/1/o12127.

[3] Isaev R.A., Podvesovskii A.G. Generalized Model of Pulse Process for Dynamic Analysis of Sylov's Fuzzy Cognitive Maps // CEUR Workshop Proceedings of the Mathematical Modeling Session at the International Conference Information Technology and Nanotechnology (MM-ITNT 2017), Vol. 1904. - P. 57-63. - DOI: 10.18287/1613-0073-20171904-57-63

[4] Podvesovskii A.G., Isaev R.A. Visualization Metaphors for Fuzzy Cognitive Maps // Scientific Visualization, 2018, Vol. 10, Num. 4, P. 13-29. - DOI: 10.26583/sv.10.4.02

[5] Zakharova, A.A., Shklyar, A.V. Informative features of data visualization tasks // Scientific Visualization, 2015, Vol. 7, Issue 2, P. 73-80.

[6] Pomerol J-C., Romero S. Multicriterion Decision in Management: Principles and Practice. - Kluwer Academic Publishers: Boston, 2000. - DOI: 10.1007/978-1-4615-4459-3.

[7] Morris M.F. Kiviat graphs: Conventions and "figures of merit" // ACM SIGMETRICS Performance Evaluation Review. - V. 3, N. 3. - P. 2-8. - New York: ACM, 1974. - DOI: 10.1145/1041691.1041692.

[8] Bondarev A.E., Kuvshinnikov A.E. Analysis of the Accuracy of OpenFOAM Solvers for the Problem of Supersonic Flow Around a Cone // ICCS 2018, Lecture Notes in Computer Science (LNCS) 10862. - P. 221-230, 2018. - DOI:10.1007/978-3-319-93713-7_18.

[9] Bondarev A., Kuvshinnikov A. Comparative Estimation of QGDFoam Solver Accuracy for Inviscid Flow Around a Cone // IEEE The Proceedings of the 2018 Ivannikov ISPRAS Open Conference (ISPRAS-2018). - P. 82-87, 2018. - DOI: 10.1109/ISPRAS.2018.00019. 